\title{
Measuring the eccentricity of GW170817 and GW190425
}

\author{
Amber K. Lenon, ${ }^{1 \star}$ Alexander H. Nitz, ${ }^{2,3}$ Duncan A. Brown ${ }^{1,4}$ \\ ${ }^{1}$ Department of Physics, Syracuse University, Syracuse NY 13244, USA \\ ${ }^{2}$ Max-Planck-Institut für Gravitationsphysik (Albert-Einstein-Institut), D-30167 Hannover, Germany \\ ${ }^{3}$ Leibniz Universität Hannover, D-30167 Hannover, Germany \\ ${ }^{4}$ Kavli Institute for Theoretical Physics, University of California, Santa Barbara, CA 93106, USA
}

Accepted XXX. Received YYY; in original form ZZZ

\begin{abstract}
Two binary neutron star mergers, GW170817 and GW190425, have been detected by Advanced LIGO and Virgo. These signals were detected by matched-filter searches that assume the star's orbit has circularized by the time their gravitational-wave emission is observable. This suggests that their eccentricity is low, but a direct measurement of their eccentricity has not yet been made. We use gravitational-wave observations to measure the eccentricity of GW170817 and GW190425. We find that the eccentricity at a gravitational-wave frequency of $10 \mathrm{~Hz}$ is $e \leq 0.024$ and $e \leq 0.048$ for GW170817 and GW190425, respectively (90\% confidence). This is consistent with the binaries being formed in the field, as such systems are expected to have circularized to $e \leq 10^{-4}$ by the time they reach the LIGO-Virgo band. Our constraint is a factor of two smaller that an estimate based on GW170817 being detected by searches that neglect eccentricity. We note that other techniques used to constrain binary neutron star eccentricity without full parameter estimation may miss degeneracies in the waveform, and that for future signals it will be important to perform full parameter estimation with accurate waveform templates.
\end{abstract}

Key words: gravitational waves - neutron stars - elliptical orbits

\section{INTRODUCTION}

The Advanced LIGO and Virgo observatories have detected two binary neutron star mergers, GW170817 (Abbott et al. 2017) and GW190425 (Abbott et al. 2020a). To date, 17 double neutron star systems have been observed through radio surveys of the Milky Way field (Martinez et al. 2017; Tauris et al. 2017; Cameron et al. 2018; Stovall et al. 2018; Lynch et al. 2018). Observations of binary neutron stars allow us to determine their formation channels (Smarr \& Blandford 1976; Canal et al. 1990; Portegies Zwart \& Yungelson 1998; Postnov \& Yungelson 2006; Kalogera et al. 2007; Kowalska et al. 2011; Tauris et al. 2017; Belczynski et al. 2018; Vigna-Gómez et al. 2018; Giacobbo \& Mapelli 2018; Mapelli \& Giacobbo 2018; Andrews \& Mandel 2019), constrain the neutron-matter equation of state (Bauswein et al. 2017; Annala et al. 2018; Fattoyev et al. 2018; De et al. 2018; Abbott et al. 2018; Capano et al. 2019; Tews et al. 2018; Most et al. 2018; Radice \& Dai 2019; Coughlin et al. 2019; Forbes et al. 2019), and test the strong-field regime of general relativity (Abbott et al. 2019).

Although the eccentricity of double neutron stars in the Milky Way field ranges from 0.06 to 0.828 (Zhu et al. 2018; Andrews \& Mandel 2019), field binaries will circularize to eccentricity $e \leq 10^{-4}$ (Peters 1964; Kowalska et al. 2011), making them detectable by matched-filter searches that neglect eccentricity (Martel

^ Email: alenon@syr.edu
\& Poisson 1999; Cokelaer \& Pathak 2009; Brown \& Zimmerman 2010; Huerta \& Brown 2013). GW170817 and GW190425 were detected by searches that neglect eccentricity (Abbott et al. 2017, 2020a), suggesting that their eccentricity is $e \lesssim 0.05$ (Huerta \& Brown 2013), however no direct measurement of their eccentricity has been made. Romero-Shaw et al. (2020) place a limit on the eccentricity of GW190425 by estimating the effect of eccentricity on the measured parameters of the signal. Here, we directly measure the eccentricity of GW170817 and GW190425 using Bayesian parameter estimation (Biwer et al. 2019).

We use the observations from the Gravitational-Wave Open Science Center (Abbott et al. 2017, 2020a), waveform templates that include eccentricity (Moore et al. 2016), and Markov Chain Monte Carlo parameter estimation (Foreman-Mackey et al. 2013; Biwer et al. 2019) to measure the eccentricity of the GW 170817 and GW190425 when they have a gravitational-wave frequency of $10 \mathrm{~Hz}$. We find that the eccentricity of GW170817 is $e \leq 0.024$ and GW190425 is $e \leq 0.048$ at $90 \%$ confidence for a uniform prior on $e$. Our limit on eccentricity of GW170817 is a factor of two smaller than the limit estimated by its detection with circular waveform templates. We note that when using a common prior on eccentricity, our limit on the eccentricity of GW190425 is a factor of three greater than the limit of Romero-Shaw et al. (2020). This is due to a degeneracy between the chirp mass and eccentricity that is not included in the analysis of Romero-Shaw et al. (2020). 
However, this difference does not invalidate their conclusions about the formation of GW190425.

Dynamical interations may form binary neutron stars with residual eccentricity, although the rate of such mergers is expected to be small in current detectors (Lee et al. 2010; Ye et al. 2019) and a search for eccentric binary neutron stars in the $\mathrm{O} 1$ and $\mathrm{O} 2$ observing runs did not yield any candidates (Nitz et al. 2019). However, since eccentricity is an interesting probe of binary formation channels and eccentric binaries may produce different electromagnetic emission than circular binary neutron stars (Radice et al. 2016; Chaurasia et al. 2018), it is important to accurately constrain the eccentricity of binary neutron stars as the number of observed mergers increases in the coming years.

\section{METHODS}

We measure the parameters of GW170817 and GW190425 using Bayseian inference (Finn 2001; Rover et al. 2006). We use gravitational-wave data from Advanced LIGO and Virgo (Blackburn et al. 2017; Abbott et al. 2020b), $\boldsymbol{d}(t)$, and a model of the gravitational waves, $H$, to calculate the posterior probability density function, $p(\boldsymbol{\theta} \mid \boldsymbol{d}(t), H)$, given by

$p(\boldsymbol{\theta} \mid \boldsymbol{d}(t), H)=\frac{p(\boldsymbol{\theta} \mid H) p(\boldsymbol{d}(t) \mid \boldsymbol{\theta}, H)}{p(\boldsymbol{d}(t) \mid H)}$,

where $\boldsymbol{\theta}$ denotes the parameters of the gravitational waveform, $p(\boldsymbol{\theta} \mid H)$, is the prior distribution on the signal parameters, and $p(\boldsymbol{d}(t) \mid \boldsymbol{\theta}, H)$, is the probability of observing the data, known as the likelihood. The likelihood models the noise in the detector as a Gaussian and depends upon a noise-weighted inner product between the gravitational waveform and gravitational-wave data, $\boldsymbol{d}(t)$. Markov Chain Monte Carlo (MCMC) techniques can be used to marginalize over the parameters to obtain the posterior probabilities (Christensen \& Meyer 2001). Our implementation of Bayesian inferences uses the $P y C B C$ Inference software package (Biwer et al. 2019; Nitz et al. 2020) and the parallel-tempered emcee sampler, emcee_pt (Foreman-Mackey et al. 2013; Vousden et al. 2016).

For GW170817 and GW190425, the MCMC is performed over the component masses of the binary, $m_{1,2}$, the component spins aligned with the orbital angular momentum, $\chi_{1,2}$, the time of coalescence, $t_{c}$, the polarization of the $\mathrm{GW}, \psi$, the inclination angle, $\iota$, and the eccentricity, $e$.

We assume a uniform prior distribution on the component masses, component spins, and coalescence time around the trigger shown in Table 2. We assume an isotropic sky location for GW190425 and a prior uniform in $\sin \iota$ for the inclination angle of both detections. We fix the sky location of GW170817 to the observed EM counterpart using a Gaussian prior distribution on the distance (Cantiello et al. 2018). We explore the prior distribution on the eccentricity by running the MCMC with two prior distributions: a prior that is uniform in $e$ and a prior uniform in $\log e$ to compare with the GW190425 results found by Romero-Shaw et al. (2020).

We use the GW strain data from the Advanced LIGO and Virgo detectors for GW170817 and GW190425, available through the LIGO Open Science Center (LOSC) (Vallisneri et al. 2015). The LOSC_CLN_4_V1 data that we use for GW170817 includes post-processing noise-subtraction performed by the LIGO/Virgo Collaboration (Blackburn et al. 2017; Driggers et al. 2019). The T1700406_v3 data that we use for GW190425 includes preprocessing glitch removal performed by the LIGO/Virgo Collaboration specifically for use in parameter estimation (Abbott et al. 2020a,b).
We high-pass the data using an eighth-order Butterworth filter with an attenuation of 0.1 at $15 \mathrm{~Hz}$. To conserve the phase of the delay, the filter is applied forward and backwards. A low-pass finite impulse response filter is applied to the data prior to resampling. The data is decimated to $2048 \mathrm{~Hz}$ for the analysis. For computing the likelihood, we use Welch's method to estimate the detector's noise power spectral density (PSD). Welch's method is used with 16 second Hanning windowed segments that are overlapped by 8 seconds. The PSD is shortened to 8 seconds in the time domain (Allen et al. 2012). The gravitational-wave data, $\boldsymbol{d}(t)$, used in the likelihood is taken from the intervals shown in Table 2. The gravitational-wave likelihood is evaluated from a low-frequency cutoff of $20 \mathrm{~Hz}$ to the Nyquist Frequency of $1024 \mathrm{~Hz}$.

A variety of waveforms are available that model eccentricity (Huerta et al. 2014; Tanay et al. 2016; Moore et al. 2016; Huerta et al. 2017; Cao \& Han 2017; Hinder et al. 2018; Tiwari et al. 2019; Moore \& Yunes 2019). From what we know of binary neutron star mergers, we expect them to have low mass, spin, and eccentricity making TaylorF2Ecc a suitable waveform. The waveform model, $\mathrm{H}$, is TaylorF2Ecc, a TaylorF2 post-Newtonian $(\mathrm{pN})$ model with eccentric corrections. We use the LIGO Algorithm Library implementation (LIGO Scientific Collaboration 2018) accurate to 3.5 $\mathrm{pN}$ order in orbital phase (Buonanno et al. 2009), $3.5 \mathrm{pN}$ order in the spin-orbit interactions (Bohé et al. 2013), $2.0 \mathrm{pN}$ order in spin-spin, quadrupole-monopole, and self-interactions of individual spins (Mikoczi et al. 2005; Arun et al. 2009), and $3.0 \mathrm{pN}$ order in eccentricity (Moore et al. 2016). Since TaylorF2Ecc follows TaylorF2 in its construction, the waveform will terminate at twice the orbital frequency of a particle at the innermost stable circular orbit of a Schwarzschild black hole.

As a check on our analysis, we estimate the parameters of GW170817 and GW190425 using two available waveforms: the TaylorF2Ecc waveform at $e=0$ and the TaylorF2 waveform. Our analyses are consistent with each other and with the parameters estimated by Advanced LIGO and Virgo (Abbott et al. 2017, 2020a).

\section{RESULTS}

We first constrain the level of the eccentricity by using the TaylorF2Ecc waveform and a prior uniform in $e$. We find that the $90 \%$ credible intervals at $10 \mathrm{~Hz}$ for GW170817 and GW190425 are $e=0.012_{-0.012}^{+0.013}$ and $e=0.025_{-0.025}^{+0.022}$ respectively. A degeneracy between the chirp mass, $\mathcal{M}$, and eccentricity, $e$ and a small correlation between the effective spin, $\chi$ eff, and $e$ are shown in our posterior distributions in Figure 1 and Figure 2. Since $\mathcal{M}$ and $\chi_{\text {eff }}$ are correlated (Baird et al. 2013; Safarzadeh et al. 2020), this will create a small correlation between $e$ and $\chi_{\text {eff }}$.

Romero-Shaw et al. (2020) estimated the eccentricity of GW190425 to determine if the formation channel was due to unstable BB mass transfer. They estimate the eccentricity induced by the supernova kick in this formation scenario to be between $10^{-6}$ and $10^{-3}$ at $10 \mathrm{~Hz}$. To find the eccentricity of GW190425, Romero-Shaw et al. (2020) reweight the posterior samples from the parameter estimation performed using circular binaries to estimate the limit of the eccentricity using the same method used to estimate the eccentricity of binary black holes (Romero-Shaw et al. 2019). They estimate the eccentricity of GW190425 at $10 \mathrm{~Hz}$ to be $e \leq 0.007$ ( $90 \%$ confidence) using a prior uniform in $\log e$. They find no evidence for or against unstable BB mass transfer as their analysis is not able to distinguish the small residual eccentricity expected from the investigated formation channel. 
Table 1. Prior distributions and GPS time intervals for GW170817 and GW190425.

\begin{tabular}{ccc}
\hline Parameters & GW170817 & GW190425 \\
\hline Component Masses $\mathrm{M}_{\odot}$ & {$[1.0,3.0]$} & {$[1.0,3.0]$} \\
\hline Component Spins & {$[-0.05,0.05]$} & {$[-0.05,0.05]$} \\
\hline Coalescence Time $(\mathrm{s})$ & {$[1187008882.33,1187008882.53]$} & {$[1240215502.917,1240215503.117]$} \\
\hline Polarization & {$[0,2 \pi]$} & {$[0,2 \pi]$} \\
\hline Inclination Angle & $\sin \iota$ & $\sin \iota$ \\
\hline Distance $(\mathrm{Mpc})$ & $40.7 \pm 2.36($ Cantiello et al. 2018) & uniform in comoving volume \\
\hline RA/Dec $\left({ }^{\circ}\right)$ & $3.44615914,-0.40808407($ Coulter et al. 2017; Soares-Santos et al. 2017) & {$[-\pi / 2, \pi / 2]$} \\
\hline Eccentricity & {$[0.0,0.1]$} & {$[0.0,0.1]$} \\
\hline \hline PSD Estimation Interval $(\mathrm{s})$ & {$[1187008382,1187008918]$} & {$[1240215313,1240215513]$} \\
\hline Likelihood Interval $(\mathrm{s})$ & {$[1187008692,1187008892]$} & \\
\hline
\end{tabular}

To more directly compare our limit on GW190425's eccentricity, we repeat our analysis using a $\log e$ prior. In Figure 3 we can see the differences in the posterior distributions of each prior. With the $\log e$ prior we estimate the eccentricity at $10 \mathrm{~Hz}$ to be $e \leq 0.023$. This is a factor of three larger than interval estimated by Romero-Shaw et al. (2020). By re-weighting the posterior samples rather than a full MCMC, the degeneracy between $\mathcal{M}$ and $e$ is missed. We find that by excluding posterior samples with lower values of $\mathcal{M}$, we can recover the upper limit reported by RomeroShaw et al. (2020). Although our limit on the eccentricity is larger than that of Romero-Shaw et al. (2020), our result does not change their conclusion: indeed the strong dependence of the eccentricity posterior on the prior seen in Figure 3 agrees with their conclusion that the signal-to-noise ratio of GW190425 is not large enough to explore the eccentricities expected in BB mass transfer. We would need to be able to determine the eccentricity at lower frequencies to distinguish the formation channel.

\section{CONCLUSION}

Our analysis used the gravitational-wave observations as well as a prior on the eccentricity to constrain the eccentricity of GW170817 and GW190425. Our $90 \%$ confidence limit using a uniform prior on $e$ for GW170817, $e \leq 0.024$, and GW190425, $e \leq 0.048$, are consistent with expectations since they were found by a circular search (Peters 1964). We have constrained the eccentricity to a factor of two smaller than estimates obtained from circular searches (Brown \& Zimmerman 2010; Huerta \& Brown 2013). Our 90\% credible intervals on the eccentricity of GW190425 are a approximately a factor of six larger than the interval estimated by Romero-Shaw et al. (2020), which used a prior uniform in $\log e$. This demonstrates the impact of prior choice, and the importance of measuring the eccentricity of signals using full parameter estimation to account for the correlation between parameters.

Unfortunately, based on current merger rate estimates the detection of an eccentric binary neutron star merger will be difficult with current observatories (Lee et al. 2010; Ye et al. 2019; Nitz et al. 2019), but gravitational-wave capture binaries that have $e \geq 0.8$ and could form in the LIGO-Virgo band (Rodriguez et al. 2018; Takátsy et al. 2019). However, since the eccentricity of the detections is expected to be low and negligible, $e \leq 0.02$, a circular search is effective in detecting them (Brown \& Zimmerman 2010; Huerta \& Brown 2013).

Current waveform models are effective for detection as spin and eccentricity are assumed to be low, but that might not be the case for future gravitational-wave signals. Future signals may have high eccentricity and spin and will need further corrections to be able to detect them efficiently and produce unbiased parameter estimates. Waveforms that better model eccentric signals will need to be developed before we are able to make a detection of a merger with a high eccentricity or spin. The detection of a binary neutron star mergers with high eccentricity or spin in future observing runs and with third generation detectors will reveal more about the formation channel of eccentric binaries and the existence of a dynamical formation channel.

Posterior samples are available in the associated data release (Lenon et al. 2020).

\section{ACKNOWLEDGEMENTS}

We acknowledge the Max Planck Gesellschaft for support and the Atlas cluster computing team at AEI Hannover. This research was supported in part by the National Science Foundation under Grant No. PHY-1748958. DAB thanks National Science Foundation Grant No. PHY-1707954 for support. AL thanks National Science Foundation Grant No. AST-1559694 for support. This research has made use of data, software and/or web tools obtained from the Gravitational Wave Open Science Center (https://www.gwopenscience.org), a service of LIGO Laboratory, the LIGO Scientific Collaboration and the Virgo Collaboration. LIGO is funded by the U.S. National Science Foundation. Virgo is funded by the French Centre National de Recherche Scientifique (CNRS), the Italian Istituto Nazionale della Fisica Nucleare (INFN) and the Dutch Nikhef, with contributions by Polish and Hungarian institutes. 


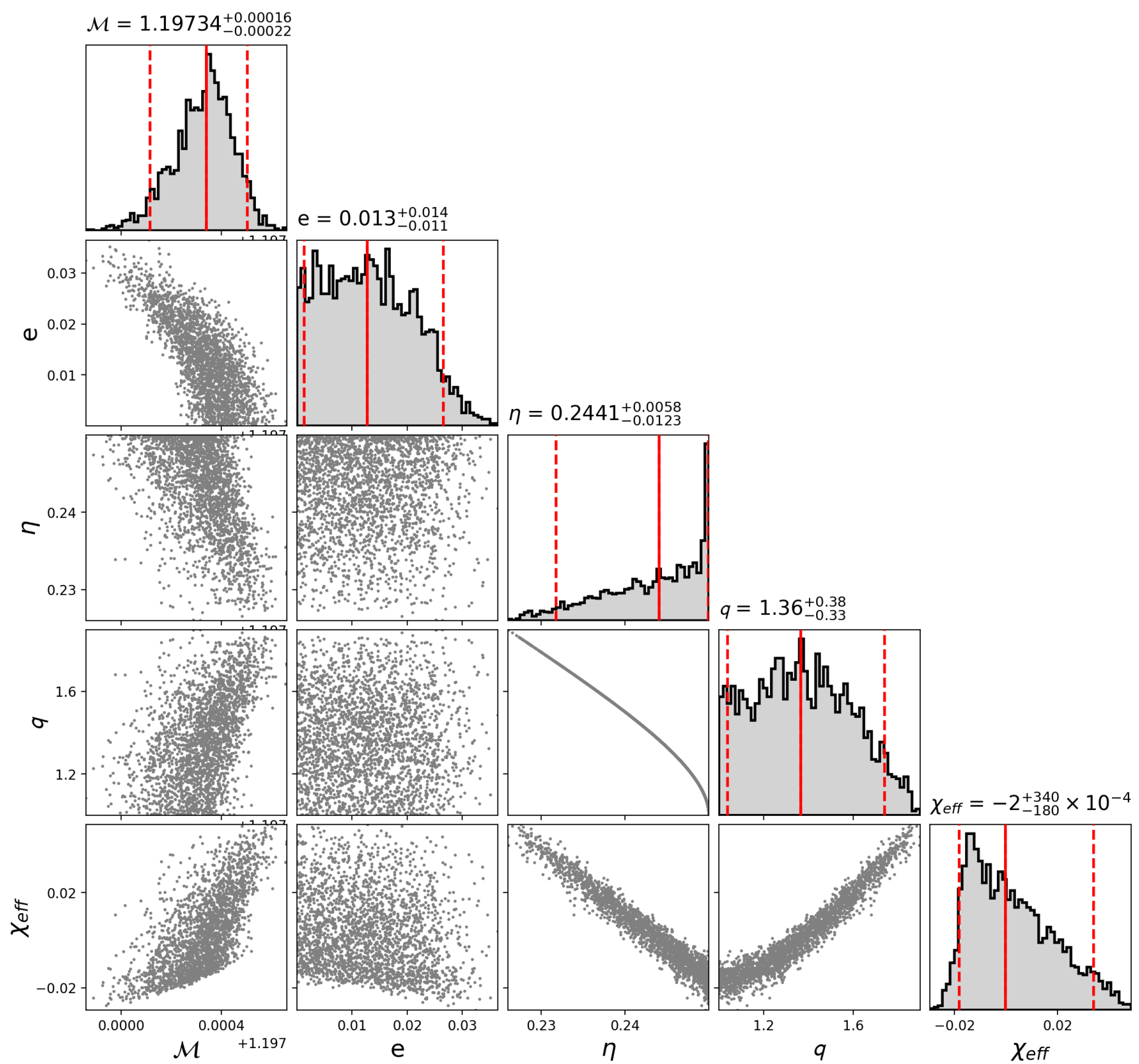

Figure 1. Posterior probability distribution of GW170817 at $10 \mathrm{~Hz}$. The analysis used a prior uniform in $e$. Each parameter is quoted with a median value (solid red line) and a $90 \%$ credible interval (dashed red lines). The chirp mass $\mathcal{M}$ is given in the detector frame. Note the degeneracy between $\mathcal{M}$ and $e$.

\section{REFERENCES}

Abbott B. P., et al., 2017, Phys. Rev. Lett., 119, 161101 Abbott B., et al., 2018, Phys. Rev. Lett., 121, 161101 Abbott B., et al., 2019, Phys. Rev. Lett., 123, 011102 Abbott B. P., et al., 2020b, Glitch model for GW190425, doi:10.7935/ggb81v94, https://dcc.ligo.org/T1900685/public

Abbott B. P., et al., 2020a

Allen B., Anderson W. G., Brady P. R., Brown D. A., Creighton J. D. E., 2012, Phys. Rev., D85, 122006

Andrews J. J., Mandel I., 2019, Astrophys. J., 880, L8

Annala E., Gorda T., Kurkela A., Vuorinen A., 2018, Phys. Rev. Lett., 120, 172703

Arun K. G., Buonanno A., Faye G., Ochsner E., 2009, Phys. Rev., D79, 104023

Baird E., Fairhurst S., Hannam M., Murphy P., 2013, Phys. Rev. D, 87,
024035

Bauswein A., Just O., Janka H.-T., Stergioulas N., 2017, Astrophys. J. Lett., 850, L34

Belczynski K., et al., 2018

Biwer C. M., Capano C. D., De S., Cabero M., Brown D. A., Nitz A. H., Raymond V., 2019, Publ. Astron. Soc. Pac., 131, 024503

Blackburn K., Weinstein A., Kanner J., Chassande-Mottin E., 2017, LOSC CLN Data Products for GW170817, doi:10.7935/K5B8566F, https: //dcc.ligo.org/LIGO-P1700349/public

Bohé A., Marsat S., Blanchet L., 2013, Class. Quant. Grav., 30, 135009

Brown D. A., Zimmerman P. J., 2010, Phys. Rev., D81, 024007

Buonanno A., Iyer B., Ochsner E., Pan Y., Sathyaprakash B. S., 2009, Phys. Rev., D80, 084043

Cameron A., et al., 2018, Mon. Not. Roy. Astron. Soc., 475, L57

Canal R., Isern J., Labay J., 1990, Ann. Rev. Astron. Astrophys., 28, 183

Cantiello M., et al., 2018, Astrophys. J. Lett., 854, L31 


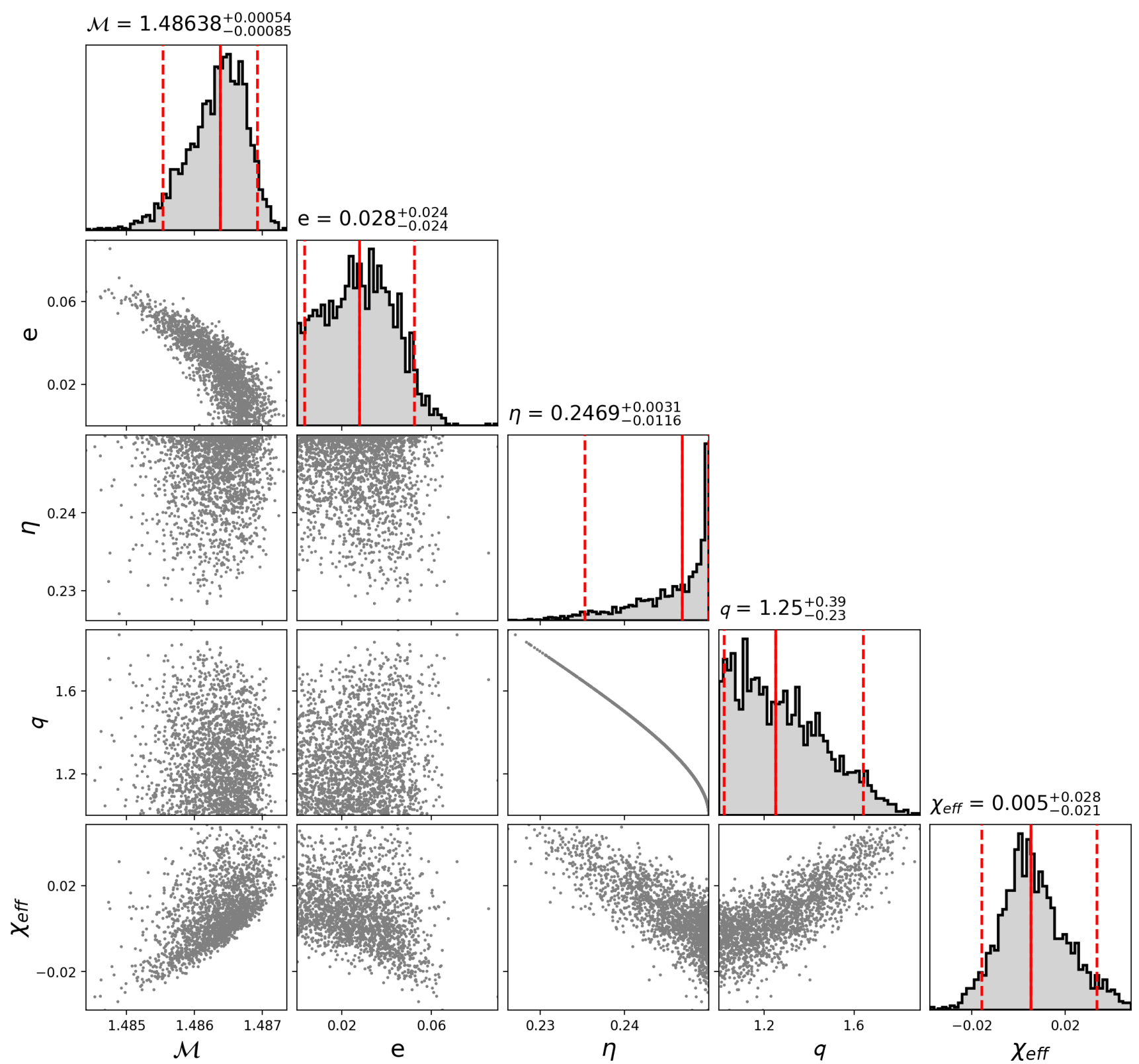

Figure 2. Posterior probability distribution of GW190425 at $10 \mathrm{~Hz}$. The analysis used a prior uniform in $e$. Each parameter is quoted with a median value (solid red line) and a $90 \%$ credible interval (dashed red lines). The chirp mass $\mathcal{M}$ is given in the detector frame. Note the degeneracy between $\mathcal{M}$ and $e$.

Cao Z., Han W.-B., 2017, Phys. Rev. D, 96, 044028

Capano C. D., et al., 2019, ] 10.1038/s41550-020-1014-6

Chaurasia S. V., Dietrich T., Johnson-McDaniel N. K., Ujevic M., Tichy W., Brügmann B., 2018, Phys. Rev. D, 98, 104005

Christensen N., Meyer R., 2001, Phys. Rev. D, 64, 022001

Cokelaer T., Pathak D., 2009, Class. Quant. Grav., 26, 045013

Coughlin M. W., Dietrich T., Margalit B., Metzger B. D., 2019, Mon. Not. Roy. Astron. Soc., 489, L91

Coulter D., et al., 2017, Science, 358, 1556

De S., Finstad D., Lattimer J. M., Brown D. A., Berger E., Biwer C. M., 2018, Phys. Rev. Lett., 121, 091102

Driggers J. C., et al., 2019, Phys. Rev., D99, 042001

Fattoyev F., Piekarewicz J., Horowitz C., 2018, Phys. Rev. Lett., 120, 172702

Finn L. S., 2001, Phys. Rev. D, 63, 102001

Forbes M. M., Bose S., Reddy S., Zhou D., Mukherjee A., De S., 2019, Phys. Rev. D, 100, 083010
Foreman-Mackey D., Hogg D. W., Lang D., Goodman J., 2013, Publ. Astron. Soc. Pac., 125, 306

Giacobbo N., Mapelli M., 2018, Mon. Not. Roy. Astron. Soc., 480, 2011

Hinder I., Kidder L. E., Pfeiffer H. P., 2018, Phys. Rev. D, 98, 044015

Huerta E. A., Brown D. A., 2013, Phys. Rev., D87, 127501

Huerta E. A., Kumar P., McWilliams S. T., O’Shaughnessy R., Yunes N., 2014, Phys. Rev. D, 90, 084016

Huerta E., et al., 2017, Phys. Rev. D, 95, 024038

Kalogera V., Belczynski K., Kim C., O’Shaughnessy R. W., Willems B., 2007, Phys. Rept., 442, 75

Kowalska I., Bulik T., Belczynski K., Dominik M., Gondek-Rosinska D., 2011, Astron. Astrophys., 527, A70

LIGO Scientific Collaboration 2018, LIGO Algorithm Library - LALSuite, free software (GPL), doi:10.7935/GT1W-FZ16

Lee W. H., Ramirez-Ruiz E., van de Ven G., 2010, Astrophys. J., 720, 953

Lenon A. K., Nitz A. H., Brown D. A., 2020, Data Release: Measuring the 

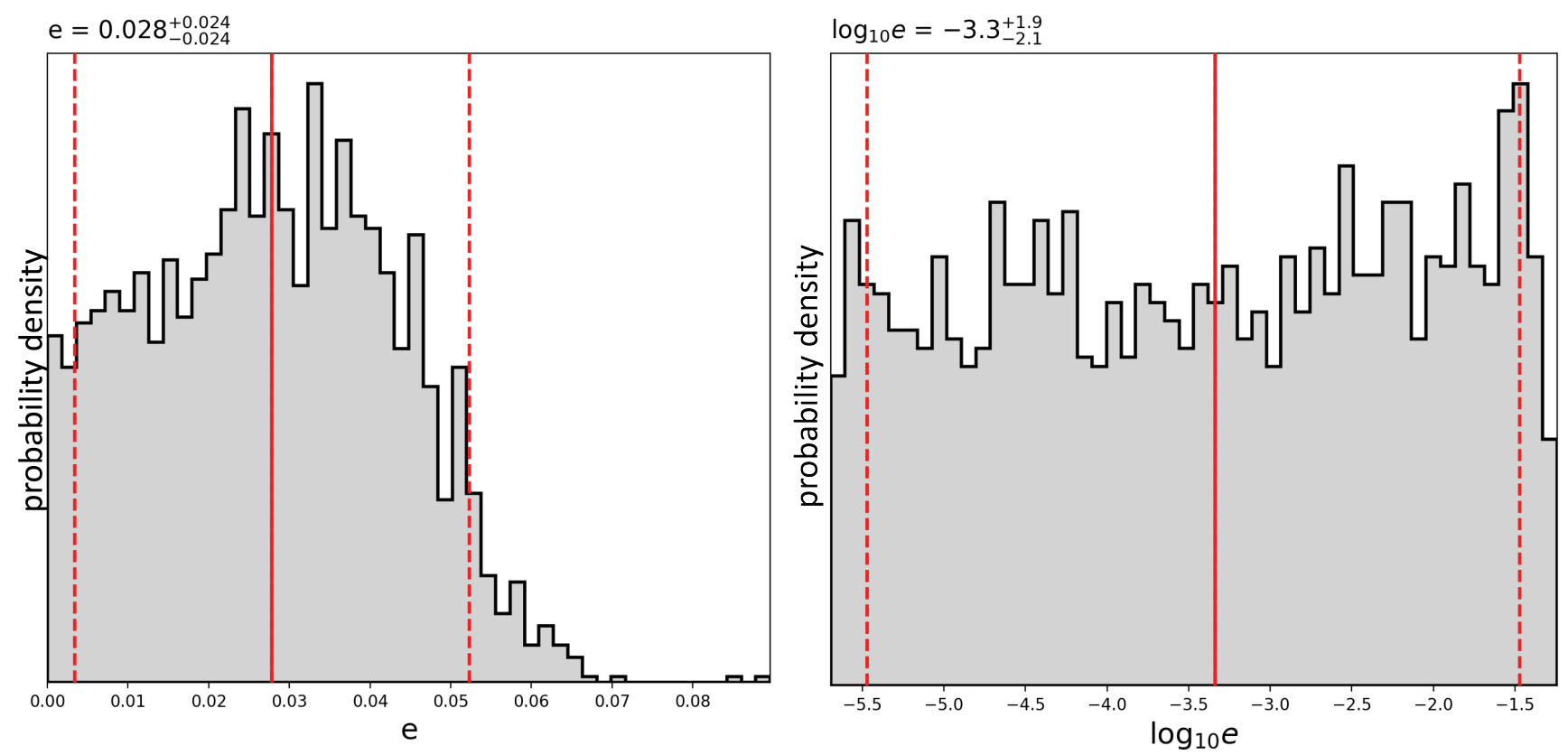

Figure 3. Eccentricity posteriors of GW190425 (solid black line) plotted against their priors (dotted line) for two choices of prior: uniform in $e$ (left) and uniform in $\log _{10}(e)$ (right). We quote the median (solid red line) and $90 \%$ credible interval (dashed red lines) for e in each posterior.

Eccentricity of GW170817 and GW190425, https://github.com/ gwastro/bns-eccentric-pe

Lynch R. S., et al., 2018, Astrophys. J., 859, 93

Mapelli M., Giacobbo N., 2018, Mon. Not. Roy. Astron. Soc., 479, 4391

Martel K., Poisson E., 1999, Phys. Rev. D, 60, 124008

Martinez J., et al., 2017, Astrophys. J. Lett., 851, L29

Mikoczi B., Vasuth M., Gergely L. A., 2005, Phys. Rev., D71, 124043

Moore B., Yunes N., 2019, Class. Quant. Grav., 36, 185003

Moore B., Favata M., Arun K. G., Mishra C. K., 2016, Phys. Rev., D93, 124061

Most E. R., Weih L. R., Rezzolla L., Schaffner-Bielich J., 2018, Phys. Rev. Lett., 120, 261103

Nitz A. H., Lenon A., Brown D. A., 2019, ] 10.3847/1538-4357/ab6611

Nitz A., et al., 2020, gwastro/pycbc: PyCBC Release v1.15.4, doi:10.5281/zenodo.3630601, https://doi.org/10.5281/ zenodo. 3630601

Peters P. C., 1964, Phys. Rev., 136, B1224

Portegies Zwart S. F., Yungelson L. R., 1998, Astron. Astrophys., 332, 173

Postnov K., Yungelson L., 2006, Living Rev. Rel., 9, 6

Radice D., Dai L., 2019, Eur. Phys. J. A, 55, 50

Radice D., Galeazzi F., Lippuner J., Roberts L. F., Ott C. D., Rezzolla L., 2016, Mon. Not. Roy. Astron. Soc., 460, 3255

Rodriguez C. L., Amaro-Seoane P., Chatterjee S., Kremer K., Rasio F. A., Samsing J., Ye C. S., Zevin M., 2018, Phys. Rev., D98, 123005

Romero-Shaw I. M., Lasky P. D., Thrane E., 2019, Mon. Not. Roy. Astron. Soc., 490, 5210

Romero-Shaw I. M., Farrow N., Stevenson S., Thrane E., Zhu X.-J., 2020, Monthly Notices of the Royal Astronomical Society: Letters

Rover C., Meyer R., Christensen N., 2006, Class. Quant. Grav., 23, 4895

Safarzadeh M., Farr W. M., Ramirez-Ruiz E., 2020

Smarr L. L., Blandford R., 1976, ApJ, 207, 574

Soares-Santos M., et al., 2017, Astrophys. J., 848, L16

Stovall K., et al., 2018, Astrophys. J. Lett., 854, L22

Takátsy J., Bécsy B., Raffai P., 2019, Mon. Not. Roy. Astron. Soc., 486, 570

Tanay S., Haney M., Gopakumar A., 2016, Phys. Rev. D, 93, 064031

Tauris T., et al., 2017, Astrophys. J., 846, 170

Tews I., Margueron J., Reddy S., 2018, Phys. Rev. C, 98, 045804

Tiwari S., Achamveedu G., Haney M., Hemantakumar P., 2019, Phys. Rev. D, 99, 124008
Vallisneri M., Kanner J., Williams R., Weinstein A., Stephens B., 2015, J. Phys. Conf. Ser., 610, 012021

Vigna-Gómez A., et al., 2018, Mon. Not. Roy. Astron. Soc., 481, 4009

Vousden W. D., Farr W. M., Mandel I., 2016, MNRAS, 455, 1919

Ye C. S., Fong W.-f., Kremer K., Rodriguez C. L., Fragione G., Rasio F. A., 2019

Zhu X., Thrane E., Oslowski S., Levin Y., Lasky P. D., 2018, Phys. Rev., D98, 043002

This paper has been typeset from a $\mathrm{T}_{\mathrm{E}} \mathrm{X} / \mathrm{LT}_{\mathrm{E}} \mathrm{X}$ file prepared by the author. 